\title{
Personality subtypes in eating disorders: validation of a classification in a naturalistic sample
}

\author{
HEATHER THOMPSON-BRENNER and DREW WESTEN
}

\author{
Background Research hasidentified \\ three personality subtypes in patients with \\ eating disorders: emotionally \\ dysregulated, constricted and high- \\ functioning/perfectionistic.
}

\begin{abstract}
Aims To see whether the subtypes are distinguished in ways indicative of valid classification, notably in patterns of adaptive functioning, comorbidity, treatment response and therapeutic interventions.
\end{abstract}

\section{Method A random sample of experienced clinicians provided data on 145 patients with bulimic symptoms, including data on eating disorder symptoms, DSM-IV comorbidity, personality pathology, treatment response and treatment interventions.}

\section{Results Patients categorised as dysregulated had the poorest functioning, most comorbidity and worst outcome, followed by patients in the constricted and high-functioning groups. The three subtypes elicited different therapeutic interventions and accounted for substantial incremental variance in outcome, holding constant the severity of eating disorder symptoms and presence of other Axis I disorders.}

\section{Conclusions The data provide} accumulating evidence for the validity of three personality subtypes in patients with eating disorders.

\section{Declaration of interest None.}

Funding detailed in Acknowledgements.
Personality pathology is common among patients with bulimia nervosa (Rossiter et $a l, 1993)$ and is often associated with poor treatment outcome (e.g. Johnson et al, 1990; Fahy \& Russell, 1993). Consistent with other research (e.g. Goldner et al, 1999), Westen \& Harnden-Fischer (2001) identified three personality subtypes that cut across eating disorder diagnoses: a dysregulated/undercontrolled pattern, characterised by emotional dysregulation and impulsivity; a constricted/overcontrolled pattern, characterised by emotional inhibition, cognitively sparse representations of the self and others, and interpersonal avoidance; and a high-functioning/perfectionistic pattern, characterised by psychological strengths alloyed with perfectionism and negative affectivity. Differences among the subtypes on aetiological and adaptive functioning variables (e.g. sexual abuse, global functioning) provided initial evidence for validity. Our study attempts to extend these findings by applying standard criteria for assessing the validity of a psychiatric classification (e.g. Robins \& Guze, 1970; see also Livesley \& Jackson, 1992) to a new sample. If the subtypes are valid and clinically relevant, they should differ in adaptive functioning, aetiological variables, patterns of comorbidity, treatment response and therapeutic interventions selected by the treating clinician.

\section{METHOD}

We used a practice network approach, in which randomly selected, experienced clinicians provide data on patients that can be aggregated across large samples (Morey, 1988; Westen \& Shedler, 1999; Shedler \& Westen, 2004). Elsewhere we have addressed in detail the rationale for clinician-reported data, including advantages and limitations (see Westen $\&$ Weinberger, 2004). The primary advantage is that clinicians are experienced observers, with skills and a normative basis with which to make inferences and recognise nuances in psychopathology. The primary objection is the possibility of bias in clinical judgement. Research suggests, however, that clinicians tend to make highly reliable and valid judgements if their observations are quantified using psychometric instruments. Correlations between treating clinicians' and independent interviewers' assessments of a range of clinical variables on measures designed for use by experienced clinicians tend to be large (typically over 0.50; Westen \& Muderrisoglu, 2003), and clinician-reported personality data predict measures of adaptive functioning, attachment patterns, and family and developmental history suggestive of validity (Nakash-Eisikovits et al, 2002; Westen et al, 2003). Clinicians' theoretical orientation predicts little variance in descriptions of clinical phenomena when they are asked to describe a specific patient rather than their beliefs or theories (Shedler \& Westen, 2004).

\section{Participants}

We contacted a random national sample of doctoral-level (MD and PhD) members of the American Psychiatric Association and American Psychological Association, with a minimum of 5 years' experience postresidency or licensure, and asked them if they would participate (uncompensated) in a study of the treatment of bulimia nervosa.

\section{Measures}

We asked participating clinicians to select their most recently terminated course of psychotherapy of three sessions or more with a female patient who had 'clinically significant symptoms of bulimia' and was binge eating and purging at the time she began treatment. We chose to include subthreshold cases to maximise generalisability to patients treated in the community. We explicitly instructed clinicians not to choose a case based on outcome, to sample both successful and unsuccessful cases (as seen below, this goal was successful). The questionnaire required $20-30 \mathrm{~min}$ to complete and included five sections.

\section{Demographics}

The first section of the questionnaire assessed clinician and patient demographic characteristics. Because prior studies using similar methods (e.g. Westen \& Shedler, 
1999) have found that the majority of clinicians report a cognitive-behavioural, psychodynamic or eclectic theoretical orientation, we measured self-reported treatment orientation by asking clinicians to tick one of four boxes: 'CBT' (cognitive-behavioural therapy), 'psychodynamic', 'eclectic' or 'other'. Those who ticked 'eclectic' were asked to describe the primary orientation that informed their work. For data-analytic purposes we created a dichotomous variable, coded 1 for 'psychodynamic spectrum' (including 'psychodynamic' and 'eclectic - primarily psychodynamic') and 2 for CBT spectrum (including 'CBT' and 'eclectic-primarily CBT'); this variable included $71 \%$ of the sample $(n=103)$. For validity of these ratings, see ThompsonBrenner \& Westen (2005).

\section{Diagnostic information}

The second section addressed patient diagnosis and adaptive functioning. Clinicians rated individual criteria for each of the DSM-IV eating disorders (American Psychiatric Association, 1994), which allowed us to apply DSM-IV diagnostic algorithms to make structured diagnoses, rather than relying on potentially unsystematic clinician use of diagnostic categories. Clinicians also rated history of eating disorder symptoms and adaptive functioning variables, such as history of psychiatric hospital admissions and ratings of Global Assessment of Functioning (GAF; American Psychiatric Association, 1994). In addition, clinicians indicated the presence or absence of DSM-IV Axis I disorders commonly comorbid with eating disorders and the ten DSM-IV Axis II diagnoses in checklist form. Respondents also rated a list of 17 'sub-threshold' personality problems used in prior research (Westen, 1997; Westen \& Arkowitz-Westen, 1998) that have been strongly associated with treatment outcome in naturalistic samples of patients with mood and anxiety disorders (Novotny et al, 2005).

\section{Personality prototype ratings}

The third section directed clinicians to rate the patient on the three personality subtypes - dysregulated, constricted and high-functioning/perfectionistic. For this study we developed paragraph-long descriptions of the prototype for each category, using the 15 items most descriptive of each from the original derivation study. To obtain both dimensional and categorical measures of these variables, we asked respondents first to rate the degree to which their patient's personality matched each prototype using a scale of $1-5$ and then to indicate which prototype best matched the patient's personality. Although similar single-item prototype ratings have yielded valid data in other domains, such as attachment and personality disorders (Mickelson et al, 1997; Westen \& Bradley, 2005), we applied principal components analysis to personality data in this study to maximise reliability of dimensional assessment.

\section{Treatment outcome}

The fourth section requested clinicians to describe the length and outcome of the treatment. It included both inferential ratings (e.g. degree of improvement in eating symptoms and degree of global improvement, rated on a scale of 1-5) and relatively objective assessments (e.g. complete remission of binge eating and purging, rated no/yes). We relied primarily on two composite scores: eating disorder outcome (two items, coefficient $\alpha=0.89$ ) and global outcome (six items, coefficient $\alpha=0.88$ ).

\section{Therapeutic interventions}

The final section directed clinicians to describe the characteristic interventions used in the treatment. We devised an adaptation for eating disorders of the Comparative Psychotherapy Process Scale (CPPS; Blagys \& Hilsenroth, 2000; Hilsenroth et al, 2003), a 20-item measure designed to assess therapy practices that have empirically differentiated psychodynamic and cognitive-behavioural treatments in controlled trials. Factor analysis of the CPPS yields a cognitivebehavioural factor and a psychodynamic factor. To maximise relevance to bulimia nervosa, we modified the CPPS to assess interventions from the CBT manual for this disorder (Fairburn et al, 1993), relevant psychodynamic interventions not addressed in the original item set, and interventions commonly employed for particular personality problems of relevance to patients with eating disorders (e.g. addressing emotional dysregulation; see Linehan, 1993). The adapted questionnaire, the CPPS-BN, has 41 items, each rated on a five-point scale.

Factor analysis of the CPPS-BN identified three factors that were robust across different factor solutions and estimation procedures, showed minimal cross-factor loadings, and accounted for $5 \%$ of the variance: 'psychodynamic', 'cognitivebehavioural' and 'adjunctive treatments' (Thompson-Brenner \& Westen, 2005). The psychodynamic factor included seven interventions identified by Blagys \& Hilsenroth (2000) as characteristic of psychodynamic therapies (e.g. addressing the patient's avoidance of important topics and shifts in mood), as well as several items we had added to reflect the broad spectrum of psychodynamic interventions used in the community (e.g. use of the therapeutic relationship for a corrective emotional experience). The cognitive-behavioural factor included seven items identified by Hilsenroth et al (2003) as characteristic of this form of therapy (e.g. teaching the patient specific techniques for coping with her symptoms) and four items we added based on the manual by Fairburn et al, (1993) (e.g. prescribing regular eating patterns). The adjunctive treatments factor included interventions such as psychiatric hospital admission not specific to any single theoretical approach. Reliabilities (coefficient $\alpha$ ) were 0.91 (15 items), 0.86 (11 items) and 0.67 (5 items), respectively. Supporting convergent and discriminant validity, self-reported CBT-spectrum and psychodynamic-spectrum clinicians significantly differed as expected on the first two factors but not on the third.

\section{Data analysis}

Because we assessed many variables with measures adapted from other studies, where possible we performed multiple validity checks and aggregated items to maximise reliability. We first used analysis of variance (ANOVA) to compare patients assigned categorically to the three prototypes on three sets of variables: adaptive functioning, aetiology and comorbidity. To compare rates of comorbid Axis I and Axis II diagnoses, we made a priori predictions using contrast analysis regarding the relative frequency of each diagnosis for patients assigned to each prototype (see Rosnow et al, 2000). (We report the results here of categorical analyses, for ease of interpretation. For the remainder of the analyses, we relied primarily on dimensional personality diagnosis to maximise power, although dimensional and categorical analyses yielded comparable data in all analyses.) Next, prior to conducting dimensional analyses of the relation between our 
personality and treatment variables, we applied principal components analysis to all personality variables included in the data-set (Axis II, sub-threshold ratings and three prototype ratings). Our twin goals were to maximise reliability of dimensional prototype diagnosis and to see if we could reproduce the dysregulated and constricted personality dimensions using a different item set and statistical procedure from that used in the original study. We then applied correlational analyses to these dimensional data to examine the relation between personality factors and treatment length and outcome. We followed this with a hierarchical multiple regression predicting outcome variables from personality factor scores, holding constant Axis I (including eating disorder) diagnoses. Finally, reasoning that clinicians should respond to very different kinds of patients with different interventions, we examined the relation between personality factors and the interventions clinicians reported using on the CPPS-BN.

\section{RESULTS}

Respondents ( $n=145)$ were evenly distributed in terms of theoretical orientation, with $37 \%$ of the sample describing their theoretical orientation as ' $\mathrm{CBT}$ ' or 'eclectic-primarily CBT', $34 \%$ as 'psychodynamic' or 'eclectic - primarily psychodynamic' and $29 \%$ as purely eclectic or 'other'. The mean duration of clinician experience was 16.1 years (s.d.=7.9). The 145 patients, nine-tenths of whom were White, averaged 28.5 years of age (s.d. $=10.2)$, with $17 \%$ rated as poor or working class, $46 \%$ as middle class, $31 \%$ as upper middle class and $6 \%$ as upper class. Thus, consistent with other studies and with the nature of the population of patients with eating disorders in the USA, the majority were White and middle class. More than two-thirds $(72 \%)$ met criteria for bulimia nervosa, purging type, at the beginning of treatment; $14 \%$ bulimia nervosa, non-purging type; $6 \%$ anorexia nervosa, binge/purging; and $8 \%$ eating disorder not otherwise specified. Thus, almost $90 \%$ of the sample $(n=125)$ met DSM-IV criteria for bulimia nervosa. The average number of binges and purges per week at the beginning of the treatment was 4.6 (s.d.=2.2) and $4.2 \quad$ (s.d.=2.8), respectively. Half of the patients engaged in excessive exercise (mean 5.7 times per week, s.d. $=2.7)$, over a third $(39 \%)$ were taking laxatives (mean 4.4 times per week, s.d. $=4.4)$ and over a half $(56 \%)$ were fasting (mean 4.1 days per week, s.d.=2.2). With respect to lifetime diagnoses, and consistent with data on eating disorder diagnostic 'crossover' using structured interviews (Eddy et al, 2002), clinicians reported that $38 \%$ of the patients had met criteria for the diagnosis of anorexia nervosa at some point. Mean pre-treatment GAF score was 51.5 (s.d.=12.3). Forty-two per cent had had at least one admission to psychiatric hospital; among these patients, the average number of periods of hospitalisation was $2.7(\mathrm{~s} . \mathrm{d} .=2.5)$. Thus, the sample showed substantial impairment.

\section{Predicting adaptive functioning, comorbidity and aetiology}

When forced to make a categorical judgement about personality subtype, clinicians classified $42 \%$ of patients as high-functioning perfectionistic, $31 \%$ as constricted and $27 \%$ as dysregulated. Approximately $84 \%$ of the sample could be categorised as strongly resembling one of the personality prototypes (rated 4 or 5 on a five-point scale). The three groups differed systematically in adaptive functioning. Pre-treatment GAF scores were highest for high-functioning patients (mean 55.9, s.d.=11.6), followed by the constricted group (mean 52.0, s.d. $=12.8$ ) and then by the dysregulated group (mean 45.3, s.d.=11.1); $F(2,133)=8.92, P<0.001$. The three groups similarly differed in history of hospitalisations $\left(\chi^{2}(2)=10.14, P=0.006\right)$, with the highest rates in the dysregulated group $(62 \%)$, followed by the constricted $(40 \%)$ and the high-functioning (29\%) groups. The three groups also differed in clinician-reported childhood sexual abuse $\left(\chi^{2}(2)=7.08, P=0.03 ; n=135\right)$, with the highest rates in patients categorised as dysregulated (42\%), compared with $20 \%$ and $19 \%$ of the constricted and highfunctioning patients, respectively. These results largely replicated the findings of Westen \& Harnden-Fischer (2001), except that the present study did not include patients with non-purging anorexia, who are more likely to be categorised as 'constricted' and to have poor adaptive functioning.

We next examined Axis I and Axis II comorbidity, on the assumption that, other things being equal, genuinely different kinds of patients should have different patterns of comorbidity. Table 1 shows the frequencies by personality subtype of diagnoses present in at least $10 \%$ of the sample. For these analyses we dummycoded absent/present ratings of each diagnosis as 0 or 1 , so that the mean values translate to frequencies (i.e. percentage of patients diagnosed with the comorbid disorder) and can be used in contrast analyses (see Rosnow et al, 2000). We conducted one-way ANOVAs with a priori contrasts to test focal one-tailed hypotheses regarding the relative frequencies for each comorbid condition. The three groups showed distinct and predictable patterns of both Axis I and Axis II comorbidity.

\section{Dimensional personality analyses}

For the remaining analyses we used dimensional measures of personality. To maximise reliability, and to see if we could reproduce the personality dimensions previously identified using a different sample and item set, we subjected all personality variables (Axis II diagnoses, sub-threshold personality pathology items and the three personality profile ratings) to principal components analysis (further details available from the author upon request). The first two components were robust across algorithms and estimation procedures: these were dysregulation and constriction (Table 2). (Although these are technically principal components, for purposes of exposition we refer to them henceforward as factors.) Dysregulation derived by principal components analysis was strongly associated with the clinician prototype rating of the same construct $(r(142)=0.81$; $P<0.001)$; the same was true of constriction $(r(143)=0.71 ; P<0.001)$. The fact that these two factors emerged despite differences from the original derivation study in sample, items and aggregation procedures (factor analysis $v$. $Q$ factor analysis) provides compelling evidence for their validity.

\section{Personality and treatment outcome}

A valid psychiatric classification should ideally predict treatment response (Robins \& Guze, 1970). Thus, we examined the relation between dysregulation and constriction and measures of treatment outcome. To avoid inflation of means, we first examined the variables for outliers. Length of treatment had two outliers, each more than 350 sessions more than the next longest treatment. We therefore dropped these two outliers and the corresponding 
Table I Variation in frequency of Axis I and Axis II disorders as a function of personality prototype categorisation $(n=138, d . f .=136)$

\begin{tabular}{|c|c|c|c|c|c|}
\hline & I & 2 & 3 & Hypothesis' & $t$ \\
\hline & High-functioning & Constricted & Dysregulated & & \\
\hline \multicolumn{6}{|l|}{ Axis I diagnosis } \\
\hline Major depressive disorder & 31.0 & 44.2 & 62.2 & $3>2>1$ & $3.05^{* *}$ \\
\hline Panic disorder & 8.6 & 16.3 & 28.9 & $3>2>1$ & $2.66 * *$ \\
\hline Post-traumatic stress disorder & 12.1 & 18.6 & 31.6 & $3>2>1$ & $2.39 *$ \\
\hline Obsessive-compulsive disorder & 15.5 & 23.3 & 31.6 & $2>3>1$ & 1.85 \\
\hline Substance use disorder & 6.9 & 16.3 & 47.4 & $3>2>1$ & $3.89 * * *$ \\
\hline Sexual disorder & 3.4 & 18.6 & 13.5 & $2,3>1$ & $2.38 * *$ \\
\hline Dissociative disorder & 0 & 14.0 & 15.8 & $3>2>1$ & $2.76 * *$ \\
\hline Bipolar disorder & 1.7 & 2.3 & 16.2 & $3>1,2$ & $3.24^{* *}$ \\
\hline Any Axis I comorbidity & 81.0 & 95.3 & 100.0 & $3>2>1$ & $4.86^{* * *}$ \\
\hline \multicolumn{6}{|l|}{ Axis II diagnosis } \\
\hline Borderline PD & 3.4 & 16.3 & 34.2 & $3>2>1$ & $8.62 * * *$ \\
\hline Narcissistic PD & 5.2 & 14.0 & 13.2 & $3>2>1$ & 1.27 \\
\hline Histrionic PD & 1.7 & 9.3 & 34.2 & $3>2>1$ & $5.00 * * *$ \\
\hline Avoidant PD & 8.6 & 23.3 & 15.8 & $2>3>1$ & $2.04 *$ \\
\hline Obsessive-compulsive PD & 8.6 & 25.6 & 13.2 & $2>3>1$ & $2.38 *$ \\
\hline Dependent PD & 8.8 & 34.9 & 29.7 & $3>2>1$ & $2.45^{*}$ \\
\hline Any Axis II comorbidity & 29.8 & 69.0 & 80.6 & $3>2>1$ & $5.75^{* * *}$ \\
\hline
\end{tabular}

PD, personality disorder.

I. Data analysed by one-way analysis of variance using planned contrasts, with percentage of patients with the disorder (coded $0 / I)$ treated as the dependent variable. To test contrasts of the form $x>y>z$ we used the contrast coefficients $I, 0,-I$. To test contrasts of the form $x>y, z$ we used the contrast coefficients 2 , $-I$, $-I$.

$* P<0.05, * * P<0.01, * * * P<0.001$, one-tailed.

Table 2 Principal components analysis of personality variables $(n=145)$

\begin{tabular}{|c|c|}
\hline Item & Loading \\
\hline \multicolumn{2}{|l|}{ Factor I: Dysregulated } \\
\hline Dysregulated prototype rating & 0.81 \\
\hline Borderline personality disorder & 0.67 \\
\hline Problems getting along with others at work & 0.67 \\
\hline Problems with impulsivity & 0.66 \\
\hline Difficulty regulating self-esteem & 0.60 \\
\hline Achievements far below level of abilities & 0.57 \\
\hline Problems with authority figures & 0.55 \\
\hline High-functioning prototype rating & -0.52 \\
\hline Problems with feeling abandoned or neglected & 0.50 \\
\hline Histrionic personality disorder & 0.48 \\
\hline Depressive (whether or not Axis I disorder) & 0.41 \\
\hline \multicolumn{2}{|l|}{ Factor 2: Constricted } \\
\hline Constricted prototype rating & 0.71 \\
\hline Problems with being emotionally constricted & 0.59 \\
\hline Problems with being rigid & 0.57 \\
\hline Dependent personality disorder & 0.54 \\
\hline Avoidant personality disorder & 0.51 \\
\hline Obsessive-compulsive personality disorder & 0.49 \\
\hline Shyness & 0.46 \\
\hline Narcissistic personality disorder & $-0.4 I$ \\
\hline
\end{tabular}

cases with the two smallest values. Length of time to recovery had one outlier, 200 sessions after the next data point; we therefore removed the highest and lowest values for this variable. Length of time to improvement had no evident outliers. As predicted, both dysregulation and constriction were positively associated with treatment length and negatively associated with outcome (Table 3). Secondary categorical analyses are illustrative here: patients rated as dysregulated attained recovery on average after 92 weeks of treatment, in comparison with 73 weeks for those rated as constricted and 51 weeks for high-functioning patients. Only $43 \%$ of the dysregulated group recovered, compared with $50 \%$ of constricted and $62 \%$ of high-functioning patients.

If the classificatory distinction we are proposing is valid, it should show incremental validity in predicting treatment response, above and beyond eating disorder symptoms (and comorbid Axis I pathology). To test this, we used hierarchical multiple regression. In the first block, we entered frequency of bingeing, frequency of purging and a composite (additive) Axis I comorbidity variable that 
Table 3 Correlations between personality factors (dimensions) and treatment outcome variables

\begin{tabular}{lccc}
\hline Outcome variable & Dysregulated & Constricted & $n$ \\
\hline Length of treatment & $0.27^{* *}$ & 0.02 & 127 \\
Weeks to improvement in eating disorder symptoms & $0.30^{* * *}$ & 0.08 & 120 \\
Weeks to recovery from symptoms & $0.41^{* * *}$ & -0.01 & 65 \\
Clinical change in symptoms (no/yes) & $-0.25^{* *}$ & -0.13 & 134 \\
Recovery from symptoms (no/yes) & -0.17 & -0.14 & 129 \\
Eating disorder outcome rating & $-0.26^{* *}$ & $-0.23^{* *}$ & 135 \\
Global outcome rating & $-0.24^{* *}$ & $-0.19^{*}$ & 135 \\
Post-treatment GAF rating & $-0.29 * * *$ & -0.11 & 133 \\
\hline
\end{tabular}

GAF, Global Assessment of Functioning.

$* P<0.05,{ }^{* * P}<0.01$, ${ }^{* * * P}<0.001$, two-tailed.

indexed the presence of the three Axis I diagnoses that consistently predicted negative outcome in zero-order correlations (major depression, panic disorder and substance use disorders). In the second block, we entered dysregulated and constricted factor scores. Table 4 reports the results for two composite criterion variables: eating disorder outcome (the extent to which the patient's symptoms improved) and global outcome. This represents a very conservative test of the validity of the personality dimensions, given that it holds constant the severity of eating pathology and Axis I diagnoses that may reflect in part personality processes.

As the data in Table 4 illustrate, adding the two personality variables in the second step substantially improved prediction of both global outcome and eating outcome. In secondary analyses, we ran these analyses again separately by theoretical orientation of the clinician (selfreported psychodynamic spectrum or CBT spectrum). Whereas outcome was not significantly associated with personality (or any other variable) in dynamic therapies, dysregulation and constriction were strongly predictive of both global and eating disorder outcome in selfreported cognitive-behavioural therapies. For global outcome, standardised $\beta=$ -0.42 for dysregulation $(P=0.004)$ and $\beta=-0.38$ for constriction $(P=0.008)$; for eating disorder outcome, standardised $\beta=-0.45 \quad(P=0.003) \quad$ and $\quad \beta=-0.30$ $(P=0.03)$, respectively.

\section{Personality and treatment interventions}

In a final set of analyses we examined the relation between clinician-reported interventions on the CPPS-BN and the dysregulated and constricted personality subtypes. Because the self-reported CBTspectrum and psychodynamic-spectrum clinicians differed substantially in the interventions they reported using, we conducted separate analyses for each clinical orientation.

Table 5 sets out the correlations between dysregulation and interventions endorsed by CBT-spectrum and psychodynamic-spectrum clinicians. As might be expected, both groups of clinicians used more adjunctive interventions and tended to address traumatic experiences with more dysregulated patients (who were more likely to have trauma histories). The most striking finding, however, was the large correlation between dysregulation and the use of psychodynamic interventions by the CBT-spectrum clinicians. Put another way, the more dysregulated the patient, the more CBT-spectrum clinicians turn to techniques designed to address personality diatheses.

Table 6 reports correlations between constriction and intervention strategies. As with dysregulation, CBT-spectrum clinicians tended to become more psychodynamic in their interventions with more constricted patients, although the effect

Table 4 Hierarchical multiple regression analyses predicting treatment outcome from eating disorder severity, Axis I comorbidity and personality factors (d.f. $=3$, 125)

\begin{tabular}{|c|c|c|c|c|c|}
\hline Outcome variable & $R$ & $R^{2}$ & Standardised $\beta$ & $F$ change (model) or $t(\beta)$ & $P$ \\
\hline \multicolumn{6}{|l|}{ Global outcome } \\
\hline Model I (eating disorder and Axis I comorbidity) & 0.30 & 0.09 & & 4.27 & 0.007 \\
\hline Model 2 (all predictors) & 0.43 & 0.18 & & 6.79 & 0.002 \\
\hline Frequency of bingeing & & & -0.06 & -0.59 & 0.55 \\
\hline Frequency of purging & & & 0.13 & 1.33 & 0.19 \\
\hline Axis I composite variable' & & & -0.14 & -1.50 & 0.14 \\
\hline Dysregulation & & & -0.22 & -2.41 & 0.017 \\
\hline Constriction & & & -0.26 & -2.98 & 0.004 \\
\hline \multicolumn{6}{|l|}{ Eating disorder outcome rating } \\
\hline Model I (eating disorder and Axis I comorbidity) & 0.34 & 0.11 & & 5.26 & 0.002 \\
\hline Model 2 (all predictors) & 0.42 & 0.17 & & 4.50 & 0.013 \\
\hline Frequency of bingeing & & & -0.06 & -0.54 & 0.59 \\
\hline Frequency of purging & & & 0.12 & 1.26 & 0.21 \\
\hline Axis I composite variable' & & & -0.20 & -2.14 & 0.034 \\
\hline Dysregulation & & & -0.18 & -2.00 & 0.048 \\
\hline Constriction & & & -0.21 & -2.40 & 0.018 \\
\hline
\end{tabular}

I. Additive variable: major depression, panic disorder and substance use disorder. 


\begin{tabular}{|c|c|c|}
\hline \multirow[t]{2}{*}{ CPPS-BN factor/selected item } & \multicolumn{2}{|c|}{ Clinician orientation } \\
\hline & $\begin{array}{c}\text { Self-reported } \\
\text { CBT spectrum } \\
(n=52)\end{array}$ & $\begin{array}{c}\text { Self-reported } \\
\text { psychodynamic spectrum } \\
(n=49)\end{array}$ \\
\hline Factor I: psychodynamic interventions & $0.46 * *$ & 0.23 \\
\hline Factor 3: adjunctive treatment factor & $0.33 *$ & $0.29 *$ \\
\hline Addressed the patient's avoidance of important topics and shifts in mood (psychodynamic factor) & $0.50 * * *$ & 0.07 \\
\hline Focused on the relationship between the therapist and patient (psychodynamic factor) & $0.30 *$ & 0.22 \\
\hline Encouraged discussion of the patient's wishes, fantasies, dreams, etc. (psychodynamic factor) & 0.26 & 0.05 \\
\hline $\begin{array}{l}\text { Focused on similarities between the patient's relationships (and perceptions of relationships) repeated } \\
\text { over time, settings, or people (psychodynamic factor) }\end{array}$ & $0.43 * *$ & 0.07 \\
\hline Helped the patient regulate intense emotions (e.g. anger, fear) (psychodynamic factor) & $0.40 * *$ & $0.40 * *$ \\
\hline Focused on ways the patient deals with anger or aggression (psychodynamic factor) & $0.47^{* *}$ & $0.43^{* *}$ \\
\hline Explored and addressed issues of sexuality (psychodynamic factor) & $0.4 I^{* *}$ & 0.12 \\
\hline $\begin{array}{l}\text { Used the therapeutic relationship to offer the patient a different model for relationships than she had } \\
\text { previously experienced (psychodynamic factor) }\end{array}$ & $0.30 *$ & 0.19 \\
\hline $\begin{array}{l}\text { Helped the patient come to terms with her relationships with and feelings about significant others from } \\
\text { the past (e.g. mother, father) (psychodynamic factor) }\end{array}$ & $0.37^{* *}$ & 0.05 \\
\hline Focused on the influence of unconscious processes on behaviour, emotions, beliefs & $0.36 * *$ & 0.25 \\
\hline Focused on the patient's conflicting feelings or desires (psychodynamic factor) & 0.21 & $0.33^{*}$ \\
\hline $\begin{array}{l}\text { Encouraged the patient to reward or punish herself for adaptive and maladaptive behaviours (e.g. giving } \\
\text { herself something when she avoided purging) }\end{array}$ & 0.24 & -0.01 \\
\hline Helped the patient problem-solve current crises or difficult interpersonal situations & $0.48^{* * *}$ & -0.04 \\
\hline $\begin{array}{l}\text { Helped the patient think of other ways to respond when she was feeling impulsive or self-destructive } \\
\text { (did not include binge eating as impulsivity/self-destructiveness) }\end{array}$ & $0.49 * * *$ & $0.29 *$ \\
\hline Encouraged patient to assert herself or get her needs met in relationships & $0.32 *$ & -0.08 \\
\hline Used conjoint in-patient or day treatment (adjunctive treatment factor) & 0.14 & $0.38 * *$ \\
\hline $\begin{array}{l}\text { Used conjoint psychosocial treatments, such as group treatment or family treatment (adjunctive } \\
\text { treatment factor) }\end{array}$ & 0.25 & -0.03 \\
\hline Used conjoint psychopharmacology (adjunctive treatment factor) & $0.28 *$ & $0.29 *$ \\
\hline
\end{tabular}

CBT, cognitive-behavioural therapy; CPPS-BN, Comparative Psychotherapy Process Scale - Bulimia Nervosa. $* P<0.05, * * P<0.01, P<0.001$, two-tailed.

was less pronounced, centring on use of the therapeutic relationship and encouraging the patient to experience and express feelings she is inhibiting. Psychodynamicspectrum clinicians, in contrast, reported becoming more cognitive-behavioural with more constricted patients, becoming more didactic and directive.

\section{DISCUSSION}

The data suggest that the population of patients treated in the community for bulimic symptoms includes a large subpopulation (approximately 60\%) with serious personality pathology. About $40 \%$ of patients in our sample had relatively high functioning accompanied by chronic problems with perfectionism and negative affectivity (e.g. anxiety, depression and self-criticism). The remainder were split equally between the dysregulated and constricted personality patterns, which appear to be robust across samples, item sets and statistical aggregation procedures. The three personality constellations, treated categorically or dimensionally, have meaningful external correlates indicative of valid diagnostic distinctions, using the criteria of Robins \& Guze (1970) and related criteria. Consistent with earlier findings (Westen \& Harnden-Fischer, 2001), patients conforming to the dysregulated subtype showed the highest rate of prior psychiatric hospitalisations (>60\%), followed by constricted (approximately $40 \%$ ) and high-functioning patients (approximately $30 \%$ ). The dysregulated group were also most likely to have a history of sexual abuse. Patients categorised as constricted in our sample were somewhat less disturbed than those in the original sample, probably because Westen \& Harnden-Fischer sampled both patients with anorexia and those with 
Table 6 Correlations between constriction and CPPS-BN factors and items

CPPS-BN factor/selected item
Clinician orientation

\begin{tabular}{cc}
\multicolumn{2}{c}{ Clinician orientation } \\
\hline Self-reported & Self-reported \\
CBT spectrum & psychodynamic spectrum \\
$(n=52)$ & $(n=49)$ \\
\hline 0.18 & -0.01 \\
-0.18 & 0.10 \\
$0.37 * *$ & 0.10 \\
$-0.31^{*}$ & $0.30^{*}$ \\
-0.08 & $0.31 *$ \\
0.15 & $-0.31 *$ \\
$0.31^{*}$ & -0.12 \\
$0.28 *$ & -0.17 \\
$0.29 *$ & 0.06 \\
$0.31^{*}$ & -0.06 \\
$0.41^{* *}$ & 0.07
\end{tabular}

Factor I: psychodynamic interventions

Factor 2: cognitive-behavioural interventions

Factor 3: adjunctive treatment factor

Taught the patient specific techniques for coping with her symptoms (CBT factor)

Actively initiated the topics of discussion and other therapeutic activities

Preferred that the patient, rather than the therapist, initiate discussion of significant issues

Encouraged the patient to experience and express feelings in the session (psychodynamic factor)

Focused on the relationship between the therapist and the patient

Established and maintained rules for therapeutic engagement

Used conjoint psychopharmacology

Used conjoint in-patient or day treatment (adjunctive treatment factor)

CBT, cognitive-behavioural therapy; CPPS-BN, Comparative Psychotherapy Process Scale - Bulimia Nervosa.

$* P<0.05, * * P<0.01, * * * P<0.001$, two-tailed.

bulimia, including severely ill patients with anorexia who are more likely to be constricted. Although the three types of patients were similar in their eating disorder symptoms $(90 \%$ sharing the bulimia nervosa diagnosis), they also showed distinct patterns of comorbidity. Patients rated as dysregulated had the highest rate of comorbid Axis I diagnoses and cluster B personality disorders. Patients rated as constricted were intermediate between the dysregulated and the highfunctioning groups on Axis I comorbidity and were particularly likely to have cluster C (avoidant, dependent and obsessivecompulsive) personality disorders.

Personality patterns also predicted differences in treatment length and outcome. Dysregulation and constriction were both negatively associated with outcome. Patients in the constricted category attained recovery on average 5 months later than the high-functioning patients, and dysregulated patients attained recovery approximately 5 months later still. The percentage of patients who recovered during treatment was lowest among the dysregulated group, followed by the constricted group, and greatest in the high-functioning patients. Of particular importance was the incremental validity of dysregulation and constriction in predicting global and eating disorder outcome above and beyond variance accounted for by eating disorder severity variables and Axis I comorbidity. Treatment response is an essential criterion laid out by Robins \& Guze (1970) for validating a diagnostic distinction, given its obvious clinical relevance.

Although the data are correlational and hence only suggestive as regards causation, clinicians appear to adjust their treatment strategies according to the patient's personality style. Cognitivebehavioural therapists report greater use of psychodynamic interventions with more dysregulated patients and a greater likelihood of using particular psychodynamic interventions with constricted patients (perhaps because of the focus of psychodynamic interventions on personality processes). Psychodynamic therapists, in contrast, increase their use of interventions associated with cognitive-behavioural therapy with more constricted patients (perhaps because patients rated highly on constriction have trouble initiating conversations and tend to have relatively empty or barren representations of themselves and others, which can make exploratory treatment more difficult). These data are striking, given that clinicians were reporting their use of interventions associated with the 'other camp'. Both groups of clinicians use significantly more interventions designed to help regulate emotions, contain impulsivity and resolve crises with more dysregulated patients.

\section{Limitations}

The major limitations of this study and the potential for bias that stems from retrospective reporting and the use of only one informant per patient (the treating clinician). These are legitimate concerns. However, several factors limit their impact. First, we attempted to minimise the bias in diagnosis and outcome by providing structured diagnostic anchors when appropriate, testing for potential biases by theoretical orientation, aggregating variables to maximise reliability, and testing hypotheses (e.g. about composite variables such as dysregulation) unfamiliar to clinicians and hence not readily biased by informant knowledge or expectancies. Second, clinicians appeared to follow our instructions to select their most recently terminated case of a patient with bulimic symptoms, rather than to cherry-pick successful cases (half of patients did not recover); they reported using a range of interventions that crossed theoretical party lines, and provided data that, in aggregate, yielded meaningful correlations with other variables that respondents could not have anticipated. However, future research should rely on multiple informants and prospective assessment.

\section{Implications}

The data have two clear implications. First, they point to the importance of assessing non-random heterogeneity among patients who share an eating disorder diagnosis. Any given research sample of patients with bulimia nervosa is likely to include subsets of patients who approximate each of the personality subtypes and hence differ on a tremendous range of variables. This may 
help to explain some of the inconsistent research findings regarding personality and eating disorders - for example, how bulimia nervosa can be associated with either borderline or obsessive-compulsive personality disorder - as well as inconsistencies in reports of the psychobiology of bulimia nervosa - for example, why some patients with the disorder show serotonin hypoactivity whereas others show the opposite pattern (Steiger et al, 2004).

Second, the treatment of personality may be integral to the effective treatment of bulimia nervosa. For a substantial subset of patients, bulimic symptoms need to be understood within the context of broader patterns of thinking, feeling, and regulating impulses and emotions. The tendency to restrict food in patients with anorexic features may be part and parcel of a constricted style of regulating impulses, emotions and so forth, just as the tendency to binge and purge in some patients with bulimia may be best understood as one of many strategies for regulating powerful emotions that outstrip their capacity to cope. Addressing these broader personality processes may require rethinking basic parameters of manualised treatments tested in randomised trials, such as their focus and brevity (see Thompson-Brenner et al, 2003; Westen et al, 2004). Dysregulation and constriction systematically predicted poorer outcome in treatment across theoretical orientations and this was particularly true for cognitive-behavioural therapy, probably because this treatment for bulimia was not designed to target personality variables. Integrative treatments, targeting both personality and eating disorder symptoms, seem particularly promising in this regard (see Westen, 2000; Thompson-Brenner \& Westen, 2005).

More speculatively, the data may have implications for genetic and behavioural genetic research on eating disorders. As Grice et al (2002) found, genetic analyses can be obstructed when phenotypes are not well identified by DSM-IV categorical diagnoses. Grice and her colleagues had difficulty linking the anorexia diagnosis to genetic markers identified in siblings concordant for the disorder but had more success identifying genetic markers of factor-analytically derived psychological traits related to the disorder. DNA was not created by committee, and empirically identified genetic loci may correspond more closely to similarly empirically derived phenotypic indicators.

\section{CLINICAL IMPLICATIONS}

- Most patients with bulimic symptoms match one of three personality prototypes: high-functioning/perfectionistic, emotionally dysregulated/undercontrolled and emotionally constricted/overcontrolled.

Personality patterns moderate treatment response, such that patients with higher ratings for dysregulation or constriction tend to take longer to treat and to fare worse in treatment, particularly treatments not designed to address personality factors.

- Treatment of personality is likely to be integral to treatment of patients with bulimic symptoms.

\section{LIMITATIONS}

- The data reflect the observations of a single informant in each case and need to be replicated with multiple observers.

- The sample is not large, reflecting the significant time commitment expected of participants for no monetary compensation.

- The data are retrospective and require replication with prospective methods.

HEATHER THOMPSON-BRENNER, PhD, Center for Anxiety and Related Disorders, Department of Psychology, Boston University, Boston, Massachusetts; DREW WESTEN, PhD, Departments of Psychology and Psychiatry and Behavioral Science, Emory University, Atlanta, Georgia, USA

Correspondence: Dr Heather Thompson-Brenner, Center for Anxiety and Related Disorders, Department of Psychology, Boston University, 648 Beacon Street, Boston, MA 02215, USA. Tel: + I 617353 9236; fax: + I 617353 9609; e-mail: htl4I@hotmail.com

(First received I3 April 2004, final revision 23 September 2004, accepted 30 September 2004)

\section{ACKNOWLEDGEMENTS}

This research was supported by a grant from the Glass Foundation. Preparation of this manuscript was supported by National Institute of Mental Health grants MH59685 and MH60892 to DW.

\section{REFERENCES}

American Psychiatric Association (1994) Diagnostic and Statistical Manual of Mental Disorders (4th edn) (DSM-IV). Washington, DC: APA.

Blagys, M. D. \& Hilsenroth, M. J. (2000) Distinctive features of short-term psychodynamic-interpersonal psychotherapy: a review of the comparative psychotherapy process literature. Clinical Psychology: Science and Practice, 7, 167-188.

Eddy, K. T., Keel, P. K., Dorer, D. J., et al (2002) Longitudinal comparison of anorexia nervosa subtypes. International Journal of Eating Disorders, 31, 191-201.

Fahy, T. A. \& Russell, G. F. M. (1993) Outcome and prognostic variables in bulimia nervosa. International Journal of Eating Disorders, 14, 135-145.
Fairburn, C. G., Marcus, M. D. \& Wilson, G.T. (1993) Cognitive-behavioral therapy for binge eating and bulimia nervosa: a comprehensive manual. In Binge Eating: Nature, Assessment and Treatment (eds C. Fairburn \& G.Wilson), pp. 361-404. New York: Guilford.

Goldner, E. M., Srikameswaran, S., Schroeder, M. L. et al (1999) Dimensional assessment of personality pathology in patients with eating disorders. Psychiatry Research, 85, $151-159$

Grice, D. E., Halmi, K. A., Fichter, M. M., et al (2002) Evidence for a susceptibility gene for anorexia nervosa on chromosome I. American Journal of Human Genetics, 70, 787-792.

Hilsenroth, M. J., Ackerman, S. J., Blagys, M. D., et al (2003) Short-term psychodynamic psychotherapy for depression: an examination of statistical, clinically significant, and technique-specific change. Journal of Nervous and Mental Disease, 19I, 349-357.

Johnson, C., Tobin, D. L. \& Dennis, A. (1990) Differences in treatment outcome between borderline and nonborderline bulimics at one-year follow-up. International Journal of Eating Disorders, 9, 617-627.

Linehan, M. (1993) Skills Training Manual for Treating Borderline Personality Disorder. New York: Guilford.

Livesley, W. J. \& Jackson, D. N. (1992) Guidelines for developing, evaluating, and revising the classification of 
personality disorders. Journal of Nervous and Mental Disease, 180, 609-618.

Mickelson, K. D., Kessler, R. C. \& Shaver, P. R. (1997) Adult attachment in a nationally representative sample. Journal of Personality and Social Psychology, 73, 1092-1106.

Morey, L. C. (1988) Personality disorders in DSM-II and DSM-III-R: convergence, coverage, and internal consistency. American Journal of Psychiatry, 145, 573-577.

Nakash-Eisikovits, O., Dutra, L. \& Westen, D. (2002) Relationship between attainment patterns and personality pathology in adolescents. Journal of the American Academy of Child and Adolescent Psychiatry, 4I, IIII-II23.

Novotny, C., Westen, D. \& Bradley, R. (2005) The external validity of efficacy trials for depression and anxiety: a naturalistic study. Psychology and Psychotherapy: Theory, Research and Practice, in press.

Robins, E. \& Guze, S. (1970) The establishment of diagnostic validity in psychiatric illness: its application to schizophrenia. American Journal of Psychiatry, 126, 983-987.

Rosnow, R. L., Rosenthal, R. \& Rubin, D. B. (2000) Contrasts and correlations in effect-size estimation. Psychological Science, II, 446-453.

Rossiter, E. M., Agras, W. S., Telch, C. F., et al (1993) Cluster B personality disorder characteristics predict outcome in the treatment of bulimia nervosa. International Journal of Eating Disorders, 13, 349-357.
Shedler, J. \& Westen, D. (2004) Dimensions of personality pathology: an alternative to the five-factor model. American Journal of Psychiatry, 161, 1743-1754.

Steiger, H., Gauvin, L., Israel, M., et al (2004) Serotonin function, personality-trait variations, and childhood abuse in women with bulimia-spectrum eating disorders. Journal of Clinical Psychiatry, 65, 830-837.

\section{Thompson-Brenner, H. \& Westen, D. (2005) A}

naturalistic study of psychotherapy for bulimia nervosa, Part I: Comorbidity and treatment outcome. Journal of Nervous and Mental Disease, in press.

Thompson-Brenner, H., Westen, D. \& Glass, S.

(2003) A multidimensional meta-analysis of psychotherapy for bulimia nervosa. Clinical Psychology: Research and Practice, 10, 269-287.

Westen, D. (1997) Divergences between clinical and research methods for assessing personality disorders: implications for research and the evolution of Axis II. American Journal of Psychiatry, 154, 895-903.

Westen, D. (2000) Integrative psychotherapy: integrating psychodynamic and cognitive-behavioral theory and technique. In Handbook of Psychological Change: Psychotherapy Processes and Practices for the 21st Century (eds C. R. Snyder \& R. Ingram), pp. 217-242. New York: Wiley.

Westen, D. \& Arkowitz-Westen, L. (1998) Limitation of Axis II in diagnosing personality pathology in clinical practice. American Journal of Psychiatry, 155, 1767-177|.
Westen, D. \& Bradley, R. (2005) Prototype diagnosis of personality. In Handbook of Personology and Psychopathology (ed. S. Strack). New York: Wiley (in press).

Westen, D. \& Harnden-Fischer, J. (200I) Personality profiles in eating disorders: rethinking the distinction between Axis I and Axis II. American Journal of Psychiatry, 158, 247-255.

Westen, D. \& Muderrisoglu, S. (2003) Assessing personality disorders using a systematic clinical interview: evaluation of an alternative to structured interviews. Journal of Personality Disorders, 17, 35I-369.

Westen, D. \& Shedler, J. (1999) Revising and assessing Axis II. Part I: Developing a clinically and empirically valid assessment method. American Journal of Psychiatry, I56, 258-272.

Westen, D. \& Weinberger, J. (2004) When clinical description becomes statistical prediction. American Psychologist, 59, 595-613.

Westen, D., Shedler, J., Durrett, C., et al (2003) Personality diagnosis in adolescence: DSM-IV Axis I| diagnoses and an empirically derived alternative. American Journal of Psychatry, 160, 952-966.

Westen, D., Novotny, C. M. \& Thompson-Brenner, H. (2004) The empirical status of empirically supported psychotherapies: assumptions, findings, and reporting in controlled clinical trials. Psychological Bulletin, 130 $631-663$. 